\title{
¿QuÉ MADERA ES BUENA PARA LEÑa? CONOCIMIENTO BOTÁNICO local en "Leñeras" del Partido de La Plata (Buenos Aires, Argentina)
}

\author{
MARÍA B. DOUMECQ ${ }^{1,2, *}$ y PATRICIA M. ARENAS ${ }^{1,2}$
}

\begin{abstract}
Resumen: El presente estudio se llevó a cabo en el partido de La Plata, Buenos Aires, Argentina. El área es diversa, con zonas urbanas y periurbanas, abundante arbolado urbano, vegetación espontánea en zonas periurbanas, mientras que en los alrededores del partido se encuentran bosques y matorrales ribereños, talares y bosques implantados. En el marco de una investigación de etnobotánica urbana, se registraron 13 sitios de venta de leña ("leñeras") y se realizaron entrevistas a los expendedores con el objetivo de relevar el conocimiento botánico local sobre las especies comercializadas, las consideradas más adecuadas, procedencia, forma de obtención, modo de reconocimiento y clasificación. Se halló un total de 32 etnotaxa comercializados como leña, entre los cuales los más vendidos son: "quebracho colorado", "itín" y "eucalipto". El principal destino de la leña es para la calefacción de hogares y, en segundo lugar, para la cocción de los alimentos. Se destaca el amplio conocimiento que posen los entrevistados acerca del tema, así como también la diversidad de prácticas relevadas en torno al uso de la leña, las estrategias de aprovisionamiento y la elevada variedad de especies utilizadas.
\end{abstract}

Palabras clave: Región rioplatense, saberes locales, comercialización, combustible, etnobotánica.

Summary: What wood is good for firewood? Local botanical knowledge in firewood stores of La Plata district, Buenos Aires (Argentina). The present study was carried out in La Plata district, Buenos Aires province, Argentina. This is a diverse area displaying urban and periurban zones, which show abundant public areas planted with trees, spontaneous vegetation in periurban aereas, while in the surroundings of La Plata district there are riparian forests and bushes, Celtis ehrenbergiana forest (called talares), and implanted forest. In the framework of an investigation of urban ethnobotany, thirteen firewood sale sites (called leñeras) were registered and the sellers were interviewed with the objective of recording their botanical knowledge about the commercialized species, those considered more suitable, origin, way of obtaining, way of recognition and classification. A total of 32 ethnotaxa were commercialized as firewood, being the most frequently sold "quebracho colorado", "itín", and "eucalipto". The main destination for firewood is for heating homes and secondly for cooking. The extensive knowledge that the interviewees have about the subject is highlighted, also the diversity of surveyed practices involved in the use of firewood, the supply strategies, and the high variety of species used.

Key words: Rio de la Plata region, local knowledge, commercialization, fuel, ethnobotany.

\section{INTRODUCCIÓN}

El presente estudio se encuadra en una línea de investigación en Etnobotánica urbana desarrollada

\footnotetext{
1 Laboratorio de Etnobotánica y Botánica Aplicada, Facultad de Ciencias Naturales y Museo, U.N.L.P. Calle $64 \mathrm{~N}^{\circ} 3$, La Plata.

2 CONICET, ARGENTINA.

*mbelendoumecq@gmail.com
}

en el Laboratorio de Etnobotánica y Botánica Aplicada (LEBA), que estudia la relación entre las personas y las plantas, sus partes y productos derivados, en los contextos pluriculturales urbanos (Hurrell, 2014; Hurrell \& Pochettino, 2014). En este marco, un tema de suma importancia es el estudio del conocimiento botánico urbano (CBU), un corpus complejo constituido por conocimientos diversos que conviven e interactúan en el mismo contexto pluricultural (Hurrell et al., 2011). 
En términos generales, los estudios etnobotánicos han prestado mayor atención a los usos de las plantas con fines alimentarios y medicinales, mientras otros han sido relegados, por ejemplo, el uso de las plantas como combustible, de relevancia en diversos contextos bioculturales. Además, en zonas urbanas y periurbanas, donde las personas prefieren utilizar los combustibles fósiles o la electricidad a medida que aumentan sus ingresos, muchos pobladores también recurren a las plantas como combustible, más por necesidad que por elección (CIFOR, 2003). En este contexto, el uso de las plantas para cocinar o generar calefacción ha sido desatendido también en los estudios de etnobotánica urbana.

La importancia del tema en estudio radica en la vigencia del mismo a nivel global, ya que la leña es un recurso que no ha caído en desuso, por el contrario, más de dos mil millones de personas utilizan leña de forma habitual para cocinar y para obtener calor en distintas partes del mundo (CIFOR, 2012). Existen numerosos estudios en Latinoamérica (Contreras-Hinojosa et al., 2003; Alves Ramos et al., 2008; Medeiros \& Albuquerque, 2011; Alves Ramos \& Albuquerque, 2012) y a nivel mundial (Marofu et al., 1997; Ogunkunle \& Oladele, 2004). Sin embargo, en la Argentina, el abordaje etnobotánico específico sobre la utilización de leña es relativamente escaso. No obstante, trabajos relevados en los últimos años evidencian que el estudio del empleo de leña es un tema emergente. Cardoso et al. (2012, 2013, 2015), Cardoso (2013), Arré et al. (2015) y Morales et al. (2017) han realizado distintos estudios sobre esta temática en la Patagonia argentina.

En la provincia de Buenos Aires se cuenta con antecedentes de estudios etnobotánicos desarrollados en el Laboratorio de Etnobotánica y Botánica Aplicada (LEBA), que incluyen la problemática de las plantas usadas como combustible en el sector meridional de la región rioplatense: talares de los partidos de Magdalena y Punta Indio (Pochettino et al., 2015; Stampella et al., 2016; Ghiani Echenique et al., 2018; Pochettino et al., en prensa). En este marco, la presente contribución es un aporte al estudio de las plantas combustibles en el ámbito de la etnobotánica urbana en la provincia de Buenos Aires. El objetivo fue relevar el conocimiento botánico local de los expendedores de leña en el partido de La Plata, las especies comercializadas, las consideradas más aptas para su uso como leña, procedencia, forma de obtención, modo de reconocimiento y clasificación.

\section{Área de estudio}

La Plata es uno de los 135 partidos de la provincia de Buenos Aires, donde la ciudad de La Plata es su cabecera y la capital provincial. El partido cuenta con 654.324 habitantes (INDEC, 2010), está situado en el noreste bonaerense y limita con los partidos de Berisso y Ensenada, al noreste, Magdalena, al sudeste, Brandsen y San Vicente al sudoeste, Berazategui y Florencio Varela, al noroeste (Fig. 1).

Este estudio se llevó a cabo entre los meses de abril de 2017 a marzo de 2018, en la ciudad de La Plata, y en las localidades de City Bell, Gonnet, Gorina, Ringuelet y Arturo Seguí. Estas últimas, son pequeñas localidades caracterizadas por la presencia de casas bajas con amplios jardines. Sus actividades económicas principales son el comercio a pequeña escala y la prestación de servicios personales y para el hogar. En la ciudad de La Plata, debido a la actividad económica y administrativa característica de una capital de provincia, transitan a diario más de un millón de personas. Su población se caracteriza por una gran cantidad de empleados administrativos, así como por un elevado número de estudiantes y empleados de la Universidad Nacional de La Plata. Hacia el este de la ciudad se encuentra una zona industrial, con un polo petroquímico de importancia e infraestructura portuaria. Hacia el sur y el oeste se ubica el "cinturón hortícola platense", una zona perirurbana con quintas o huertos que proveen verduras y frutas frescas para la región y otras zonas del país, y también floricultura (Pochettino et al., 2008; Hurrell \& Pochettino, 2014; Pochettino et al., 2014).

El partido de La Plata es un área diversa, con sectores urbanos y periurbanos con distintas historias locales de permanencia en un espacio determinado, pero con un intensa acción humana que se expresa en el avance de la urbanización. El estudio del ámbito periurbano, implica el abordaje de un territorio en situación de interfase (o ecotono) entre dos tipos de ambientes bien diferenciados: el campo y la ciudad, y la complejidad de las relaciones ecosistémicas entre ambos. Los límites del espacio periurbano son móviles, en función de los ritmos de la urbanización (Barsky, 2005, 2010; Hurrell \& Pochettino, 2014). 


\section{B. Doumecq y P. M. Arenas - Conocimiento botánico local en leñeras de La Plata}
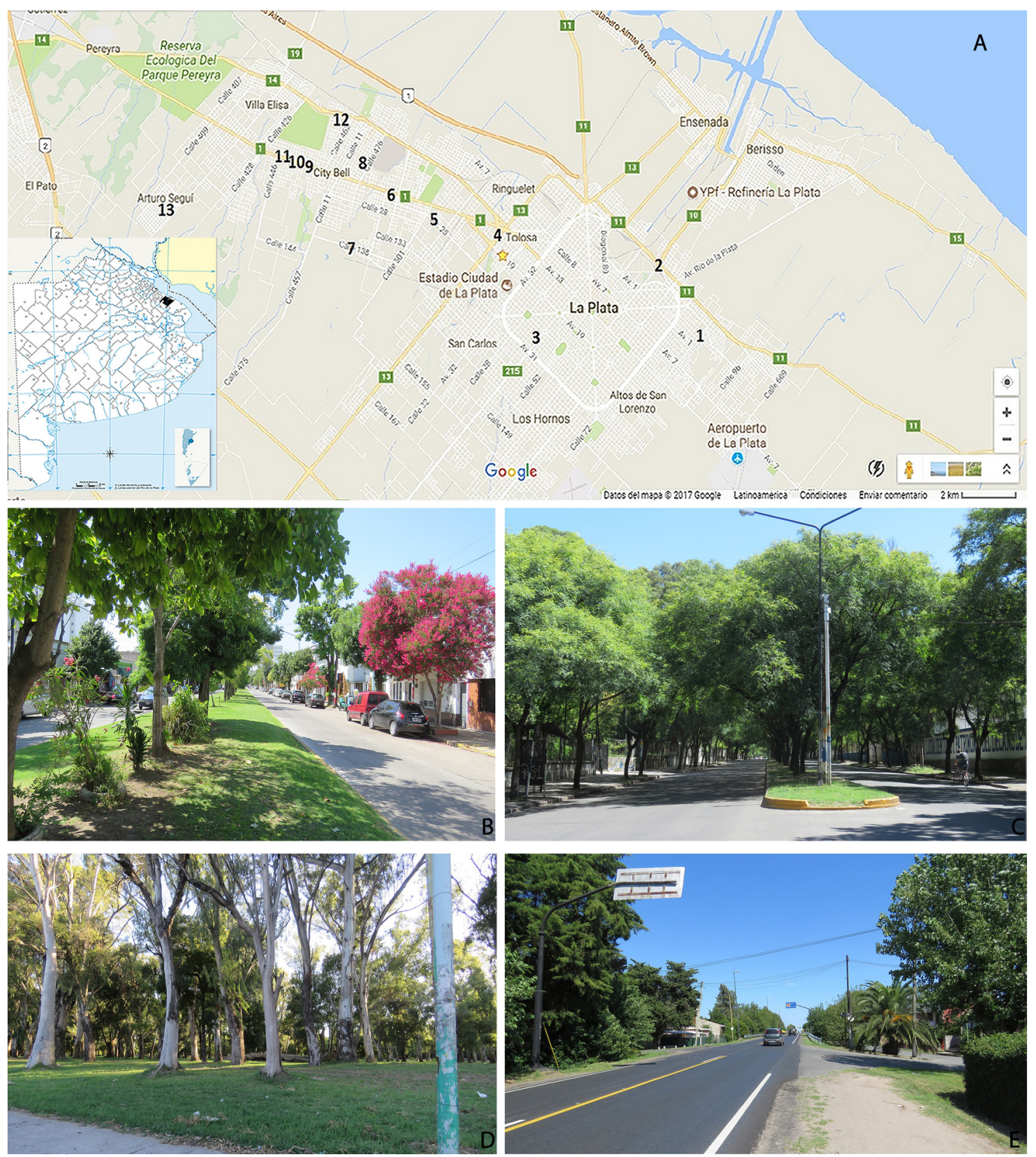

Fig. 1. Área de estudio. A: Mapa de la provincia de Buenos Aires y partido de La Plata. Derecha: detalle del partido de La Plata y leñeras relevadas; B y C: arbolado urbano de algunas avenidas del casco de la ciudad de La Plata; D: Paseo de bosque de La Plata con diversas especies del género Eucalyptus; E: vista del Camino General Belgrano y su arbolado.

En la flora urbana se encuentran diversas especies leñosas cultivadas que conforman el arbolado de calles y avenidas, y de los accesos a la ciudad de La Plata. En particular, ésta se caracteriza por la presencia de numerosas plazas y parques, además del denominado "Paseo del Bosque", que con sus 80 hectáreas constituye el espacio verde más destacado de la ciudad. El mismo se formó a 
partir de la estancia de Martín Iraola, expropiada con motivo de la fundación de La Plata, en 1882, donde se pueden encontrar diversas especies de los géneros Eucalyptus, Populus, Quercus y Salix (Delucchi et al., 1993). En los espacios entre las localidades estudiadas, distintas especies leñosas crecen de forma espontánea, como Morus alba L., "morera blanca", Gleditsia triacanthos L., "acacia negra", Manihot grahamii Hook., "falso café", Ligustrum lucidum W. T. Aiton, "ligustro" y Populus alba L., "álamo blanco" o "plateado".

En las cercanías del partido de La Plata, fuera del área de estudio, se encuentran formaciones boscosas que, por su proximidad, constituyen fuentes de aprovisionamiento de leña. Se incluyen: 1) bosques y matorrales ribereños del río de la Plata, desde el partido de Berazategui hasta el de Punta Indio, donde crecen árboles como Erythrina cristagalli L., "ceibo", Pouteria salicifolia (Spreng.) Radlk., "mata ojo", y Salix humboldtiana Willd., "sauce criollo"; 2) los talares característicos hacia el interior de la fisonomía anterior, desde el partido de San Nicolás al norte, hasta el de Mar Chiquita al sur (Parodi, 1940; Torres Robles, 2009), con elementos florísticos de la Provincia Fitogeográfica del Espinal (Cabrera, 1971; Burkart et al., 1999), con predominio de Celtis ehrenbergiana (Klotzsch) Liebm., "tala", acompañado por Scutia buxifolia Reisseck, "coronillo", Jodina rhombifolia (Hook. \& Arn.) Reissek, "sombra de toro", Schinus longifolius (Lindl.) Speg., "molle", entre otras; se trata de bosques muy degradados que han constituido la fuente principal de leña desde la época colonial (Athor, 2006; Torres Robles \& Tur, 2006); en la actualidad incluye reservas naturales como el Parque Costero del Sur, Pearson y Campos del Tuyú (Delucchi et al., 1993); 3) pastizales, más hacia el interior, mayormente reemplazados por campos de cultivo y pasturas para el ganado, en general con forestación peridoméstica, ornamental y de reparo, de especies de Eucalyptus, Populus, Salix, Pinus y Robinia entre otras, que se emplean a menudo para obtención de leña.

Es decir que, en la zona de estudio, tal como es mencionado anteriormente, existe abundante material leñoso potencialmente utilizado por los pobladores como leña; algunas de estas especies poseen maderas de mediana-alta densidad (0,8-0,9 $\mathrm{Kg} / \mathrm{dm}^{3}$ ) como el "tala", "coronillo" o el "espinillo"; otras de mediana-baja densidad $\left(0,7-0,6 \mathrm{Kg} / \mathrm{dm}^{3}\right)$ como la "acacia negra", la "acacia blanca", la "mora", el "fresno" o los "eucaliptos" y especies de baja densidad $\left(0,3-0,4 \mathrm{Kg} / \mathrm{dm}^{3}\right)$ como los "álamos" y "sauces" entre otros (Atencia, 2003).

\section{Métodos}

Se aplicaron métodos y técnicas cualitativas usuales en el trabajo etnobotánico: observación participante, entrevistas semiestructuradas, listados libres y caminatas con los entrevistados. La selección de los mismos se realizó a través de una metodología combinada, utilizando al inicio de la investigación el método "bola de nieve" (Martin, 1995; Alexíades, 1996; Bernard, 2000; Albuquerque \& Lucena, 2004; Albuquerque et al., 2014a, b), complementando con una búsqueda en Internet de sitios de expendio de leña, tratando de considerar a todos los vendedores de leña del partido y de obtener un relevamiento más exhaustivo, aunque pueden existir sitios no relevados. El método "bola de nieve" fue crucial para localizar sitios de venta informal y pequeños comercios de venta de leña que de otra manera hubiese sido muy difícil de hallar; mientras que la búsqueda por internet a través de distintos buscadores y páginas web (utilizando palabras claves tales como leñeras, leña, carbonería, venta de leña) como guías comerciales del partido complementaron la búsqueda ya que, a través de ellos, se localizaron otros comercios formales, principalmente grandes leñeras.

Se relevaron 13 sitios de expendio de leña, 12 correspondientes a comercios abiertos al público durante todo el año, y una vivienda particular donde se vende leña sólo en invierno, la cual se ha considerado como un comercio más, a pesar de su carácter informal. De ellos, 8 son exclusivos de venta de leña y carbón, y se denominan leñeras; el resto, son comercios como madereras, herrerías y verdulerías, que expenden leña como actividad secundaria. Las entrevistas fueron realizadas, previo consentimiento informado, a un expendedor por sitio de expendio. En algunos casos, la persona entrevistada era el dueño del comercio, en otros, el encargado. Los entrevistados son personas de ambos sexos, entre 25 y 70 años, todos oriundos de la zona, salvo un caso, donde el entrevistado proviene de la provincia del Chaco, pero vive en la zona desde hace más de 40 años. En las entrevistas, 


\section{B. Doumecq y P. M. Arenas - Conocimiento botánico local en leñeras de La Plata}

además de consignar los datos personales de cada entrevistado, se abordaron los siguientes temas: tiempo de trabajo en el rubro, si utiliza leña en su hogar, qué cantidad venden, a quienes, qué especies, cuáles son las más solicitadas, cuáles las mejores, por qué, cómo se clasifican, de dónde proceden, cómo se obtienen, cuáles son las limitantes para la obtención.

La información relevada durante las entrevistas se registró a través de libretas de campo y diversos medios audiovisuales (grabador, cámara fotográfica). También se obtuvieron muestras (fragmentos de leño) de las especies mencionadas por los entrevistados con la finalidad de realizar su identificación taxonómica. Las mismas fueron depositadas en las colecciones etnobotánicas del Laboratorio de Etnobotánica y Botánica Aplicada (LEBA).

Para la identificación de las especies se tuvo en cuenta el nombre local, la procedencia y la bibliografía específica (Tortorelli, 1956; Leonardis, 1977; Rivera \& Galiussi, 2015). Las muestras obtenidas se analizaron macroscópicamente con lupa de mano y, en los casos en que fue necesario, se realizaron cortes a mano alzada y se observaron al microscopio óptico. Además, las muestras problema se compararon con materiales de referencia. Para las especies propias de la zona, se recolectaron muestras de referencia de leño y para el caso de las especies que proceden del norte del país se utilizó material procedente de la xiloteca de la cátedra de Dendrología (XCD), Facultad de Ciencias Agrarias y Forestales, UNLP.

\section{Resultados}

Características de los expendedores y de los sitios de expendio

Las personas entrevistadas constituyen un grupo heterogéneo, lo que se refleja en que la inserción en la actividad leñera es reciente en algunos casos, mientras que en otros es de larga data y los conocimientos implicados fueron transmitidos de generación en generación, aunque es preciso aclarar que en algunos casos, más que una elección es una necesidad: "La venta de leña nosotros la hacemos como subsistencia, no es porque tenemos un trabajo fijo y esa plata la invertimos para vender leña, directamente nos dedicamos a eso, porque mi abuelo era leñero, mi papá empezó a trabajar de otra cosa, de golpe todos despedidos y bueno, se dedicó a la leña, no es porque mi abuelo era leñero, sino que es lo único que sabía hacer, pero ese es nuestro trabajo, después si se suma otro trabajo otra changa lo hacemos" (E. S., 25, Arturo Seguí, 2017). La leña, en los sitios de expendio, suele disponerse en dos pilas: una constituida por especies propias de la zona, las que venden mezcladas, y otra con las especies de madera más dura, que proceden principalmente, del norte del país; en ocasiones se apila también por especie (Fig. 2). Según señalan los entrevistados, a pesar de hallarse mezclada la leña de la zona, si el comprador solicita una especie en particular, esta se selecciona porque son capaces de reconocerlas. La forma de identificación es principalmente por el color de la madera, por su peso y por el tipo de corteza. Los expendedores clasifican sus leñas de acuerdo con diversos criterios: 1) según la dureza (duras, semiduras y blandas), 2) por especie, 3) según la estufa del usuario: salamandra (tamaño chico), hogar (mediana o grande), 4) por tamaño (grande, mediano y chico). 5) palitos y astillas para el asado. Combinando en la mayoría de los casos algunos de estos criterios, por ejemplo, lo más frecuentemente mencionado fue la clasificación por su dureza y además por su tamaño.

Todos los sitios relevados abastecen de leña a hogares particulares, y el $46 \%$ además lo hace a parrillas, panaderías, herrerías y otros lugares de reventa.

La forma en que se expende la leña puede ser a granel o en bolsas. Además, suelen vender bolsas "iniciadoras", es decir, las que sirven para encender el fuego, constituidas por maderas finas que provienen del reciclado de cajones de pollos o de los desechos de las madereras. La identificación de las especies que constituyen estas maderas, no fue mencionada por los entrevistados; posiblemente, la madera de los cajones corresponde a especies de Salix y Populus y la de los desechos de las madereras a especies de Pinus y Eucalyptus.

La cantidad de leña que se vende varía según su destino: si es para consumo familiar o para reventa; si se emplea para asados o para calefacción del hogar. También depende de: 1) las condiciones de temperatura en el invierno; 2) del tipo de estufa que tenga el usuario (la salamandra permite una mejor regulación de la combustión y un mejor control 
Bol. Soc. Argent. Bot. 53 (3) 2018
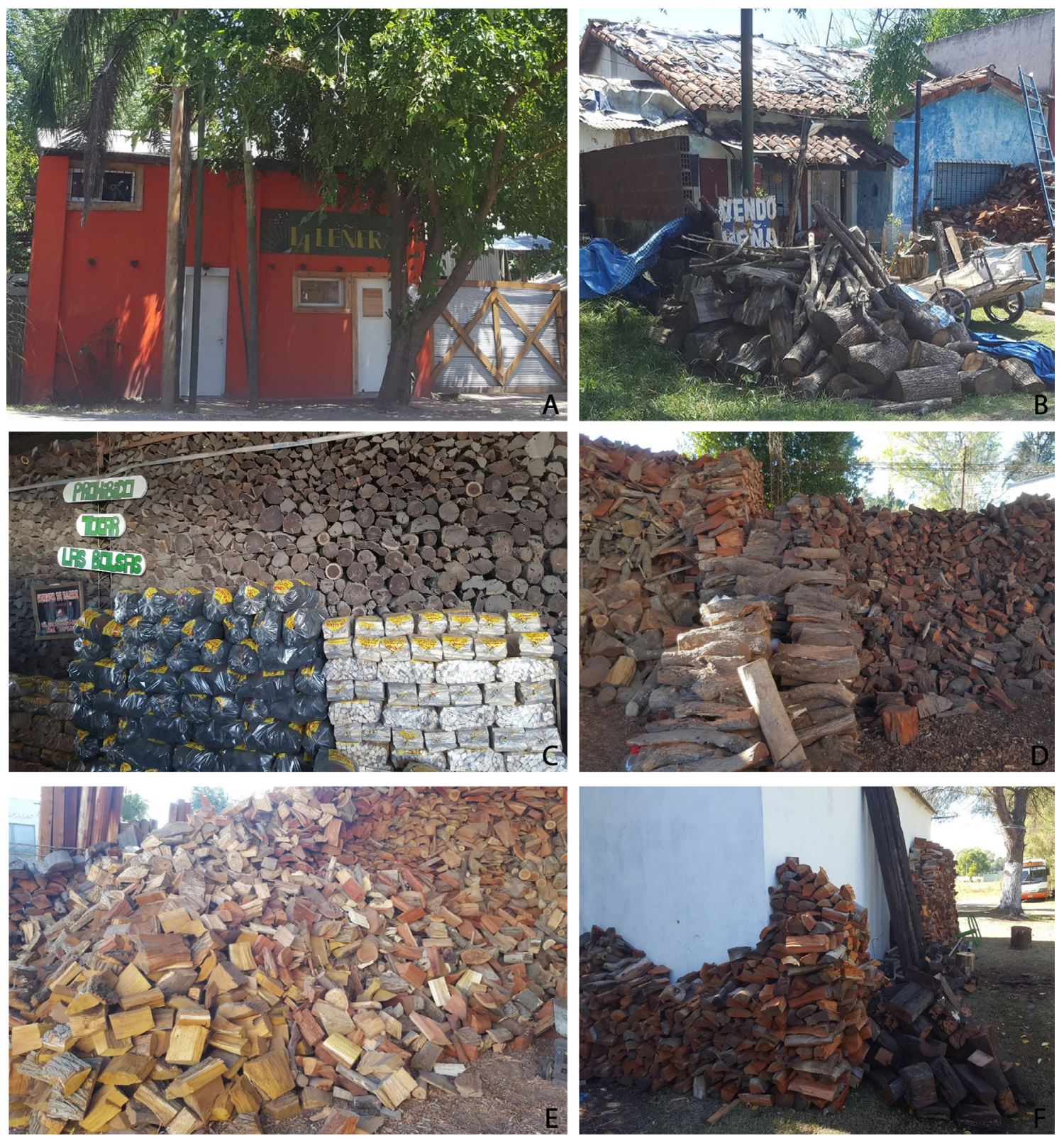

Fig. 2. Sitios de venta de leña. A: aspecto exterior de una leñera; $\mathbf{B}$ : casa particular de venta de leña a pequeña escala; C: pila de leña, carbón y bolsas "iniciadoras"; D: leña apilada por especie; de izquierda a derecha, eucalipto colorado, espinillo y quebracho colorado; E: mezcla de leña de distintas especies propias de la zona, F: pila de leña de eucalipto y quebracho y durmientes de quebracho aun sin cortar.

del humo que el hogar, por lo que también supone un ahorro de combustible); 3) de las dimensiones del ambiente a calefaccionar; si los usuarios sólo emplean leña o si suplementan la calefacción con gas o electricidad; del tiempo de encendido (todo el día o sólo por la noche); 4) de la especie utilizada (por ejemplo, el pino puede tardar unas 3 horas en quemarse; el aromo o el eucalipto, 5-6 horas). 


\section{B. Doumecq y P. M. Arenas - Conocimiento botánico local en leñeras de La Plata}

En general, los entrevistados coinciden en que, para uso familiar, una casa tipo para cuatro personas compra $250 \mathrm{~kg}$ de leña por semana, mientras que en establecimientos como las parrillas, donde tienen encendido el fuego todo el día, o las verdulerías, que revenden la leña, pueden comprar hasta $1.000 \mathrm{~kg}$ por semana.

\section{Forma de obtención de leña}

Se detectaron tres estrategias: 1) extracción y compra (el $61 \%$ de los entrevistados obtiene la leña de la zona de estudio y complementa la actividad con la compra y reventa de leña); 2) compra (el 31\% de los comercios revende la leña que compra, de modo que resulta una actividad secundaria y complementaria del rubro principal (verdulerías o madereras); 3) extracción (el $8 \%$ la obtiene de la zona). Dentro del modo "extracción" se incluye la poda de árboles de la ciudad, la limpieza de terrenos (lo que implica voltear árboles en pie y cortar), extracciones de árboles caídos. Se junta todo lo que haya a disposición, independientemente de si es considerada buena o no para leña: "Todo se vende, nada se tira" (N. 53 años, City Bell, 2017) "La mayoría de los árboles que no son de acá, que dicen que son exóticos, son plantados, que la gente te dice vení, podame el árbol, te cobramos tanto, todo sirve" (E. S., 25, Arturo Seguí, 2017). Para la extracción se utilizan diferentes herramientas: motosierra, sierra circular, hacha manual o hidráulica, retroexcavadora, balanza y diferentes vehículos para el traslado del material (autos, camionetas y camiones), donde la herramienta más mencionada es la motosierra (Fig. 3).

En cuanto a la compra de leña, los entrevistados eligen especies de madera dura, consideradas "buenas" porque generan buena brasa y calor, y duran mucho, como Schinopsis balanseae Engl., "quebracho colorado" y el "itín". Las mismas provienen de diferentes provincias del norte del país, cortadas y listas para vender, o bien recortadas manualmente con hacha para usar como leña para salamandras. Según los entrevistados, para efectuar el transporte es necesario contar con una Guía de tránsito de productos forestales, que se tramita vía online en la página web del Ministerio de Agroindustria y tiene una validez de 72 horas. Para el caso de productos provenientes de bosques nativos se debe abonar una suma determinada por tonelada y se debe declarar la cantidad transportada, el importe y los datos personales.
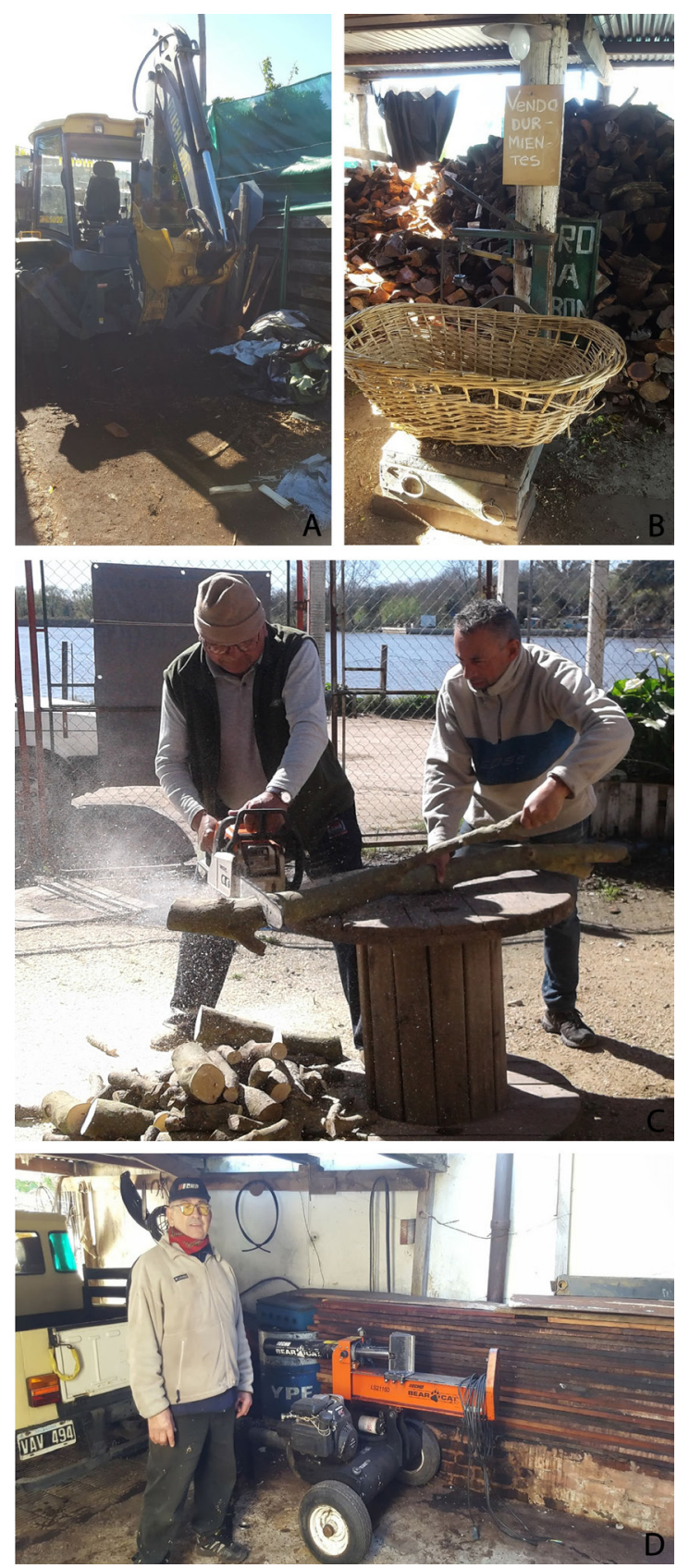

Fig. 3. Algunas herramientas utilizadas por los comerciantes. A: retroexcavadora; B: balanza; C: motosierra; D: hacha hidráulica.

\section{Época de aprovisionamiento}

La época de mayor aprovisionamiento es en el verano: "La leña tiene que estar seca, se corta en 
Bol. Soc. Argent. Bot. 53 (3) 2018

Tabla 1. Etnotaxones comercializados, origen, forma de obtención y dureza según los entrevistados.

\begin{tabular}{|c|c|c|c|c|}
\hline Etnotaxón & Nombre científico y familia Botánica & $\begin{array}{l}\text { Natival } \\
\text { Exótica* }\end{array}$ & $\begin{array}{l}\text { Forma de } \\
\text { obtención** }\end{array}$ & Dureza*** \\
\hline Acacia negra & Gleditsia triacanthos L. (Fabaceae) & Exótica & $E$ & Semidura \\
\hline Acacia & Robinia pseudoacacia L. (Fabaceae) & Exótica & $E$ & Semidura \\
\hline Álamo & Populus spp. (Salicaceae) & Exótica & $E$ & Blanda \\
\hline Algarrobo blanco & Prosopis alba Griseb. (Fabaceae) & Nativa & $\mathrm{C}$ & Dura \\
\hline Aromo & Acacia spp. (Fabaceae) & Exótica & $E$ & Semidura \\
\hline Casuarina & Casuarina cunninghamiana Miq. (Casuarinaceae) & Exótica & $E$ & Semidura \\
\hline Cedro azul & Cedrus atlantica (Endl.) Manetti ex Carrière (Pinaceae) & Exótica & $E$ & Blanda \\
\hline Ciprés & Cupressus sempervirens L. (Cupressaceae) & Exótica & $E$ & Blanda \\
\hline Espinillo & Vachellia caven (Molina) Seigler \& Ebinger, (Fabaceae) & Nativa & E y C & Semidura \\
\hline Eucalipto & Eucalyptus spp. (Myrtaceae) & Exótica & E y C & Semidura \\
\hline Eucalipto amarillo & Eucalyptus globulus Labill. (Myrtaceae) & Exótica & $E$ & Semidura \\
\hline Eucalipto blanco & Eucalyptus globulus Labill. (Myrtaceae) & Exótica & $\mathrm{E}$ & Semidura \\
\hline Eucalipto colorado & Eucalyptus camaldulensis Dehnh (Myrtaceae) & Exótica & $E$ & Semidura \\
\hline Eucalipto globulus & Eucalyptus globulus Labill. (Myrtaceae) & Exótica & $E$ & Semidura \\
\hline Eucalipto medicinal & Eucalyptus sideroxylon A.Cunn. ex Woolls (Myrtaceae) & Exótica & $E$ & Semidura \\
\hline Falso café & Manihot grahamii Hook. (Euphorbiaceae) & Nativa & $E$ & Blanda \\
\hline Fresno & Fraxinus pennsylvanica Marshall (Oleaceae) & Exótica & $E$ & Semidura \\
\hline Guayabo & Psidium guajava L. (Myrtaceae) & Nativa & $\mathrm{C}$ & Dura \\
\hline Guayacán & $\begin{array}{c}\text { Caesalpinia paraguariensis (D. } \\
\text { Parodi) Burkart (Fabaceae) }\end{array}$ & Nativa & $\mathrm{C}$ & Dura \\
\hline Itín & Prosopis kuntzei Harms (Fabaceae) & Nativa & $\mathrm{C}$ & Dura \\
\hline Laurel & Laurus nobilis L. (Lauraceae) & Exótica & $E$ & Blanda \\
\hline Ligustro & Ligustrum lucidum W.T. Aiton (Oleaceae) & Exótica & $E$ & Semidura \\
\hline Maclura & Maclura pomífera (Raf.) C.K.Schneid. (Moraceae) & Exótica & $E$ & Semidura \\
\hline Mora & Morus alba L. (Moraceae) & Exótica & $E$ & Semidura \\
\hline Paraíso & Melia azedarach L. (Meliaceae) & Exótica & $E$ & Blanda \\
\hline Pino & Pinus spp. (Pinaceae) & Exótica & $E$ & Blanda \\
\hline Piquillín & Condalia microphylla Cav. (Rhamnaceae) & Nativa & $\mathrm{C}$ & Dura \\
\hline Quebracho blanco & Aspidosperma quebracho-blanco Schltdl. (Apocynaceae) & Nativa & $\mathrm{C}$ & Dura \\
\hline Quebracho colorado & Schinopsis balanseae Engl. (Anacardiaceae) & Nativa & $\mathrm{C}$ & Dura \\
\hline Sauce común & Salix humboldtiana Willd. (Salicaceae) & Nativa & $E$ & Blanda \\
\hline Tala & Celtis ehrenbergiana (Klotzsch) Liebm. (Celtidaceae) & Nativa & $E$ & Semidura \\
\hline Vinal & Prosopis ruscifolia Griseb. (Fabaceae) & Nativa & $\mathrm{C}$ & Dura \\
\hline \multicolumn{5}{|c|}{$\begin{array}{l}\text { Referencias: } \\
\text { *En negrita resaltado las especies nativas de la Región Rioplatense } \\
{ }^{* *} \text { E: extracción de la zona; C: compra } \\
{ }^{* * *} \text { Valoración local }\end{array}$} \\
\hline
\end{tabular}




\section{B. Doumecq y P. M. Arenas - Conocimiento botánico local en leñeras de La Plata}

verano. Para secar el eucalipto ¿sabes cómo se hace? Se lo riega en verano todos los días y se lo deja al sol, así se seca el agua y sale la savia de adentro" (H. D. T, 67 años, masculino. City Bell, 2017). "En sí la leña se consigue en verano, los desmontes son en verano, y las podas en invierno, si vos querés conseguir leña te conviene en verano" (E. S., 25 años, masculino. Arturo Seguí, 2017).

\section{Etnotaxones relevados}

En total, se registraron 32 etnotaxones comercializados como leña, de estos, sólo 2, Prosopis kuntzei Harms, "itín” y Aspidosperma quebracho-blanco Schltdl., "quebracho blanco", se venden además como carbón. Las familias mejor representadas son Fabaceae y Myrtaceae. Del total relevado, 12 etnotaxones son nativos de diferentes zonas de la Argentina siendo solo 4 de ellas nativas de la zona en estudio y 20 exóticos. Además, 22 de estos se obtienen por extracción dentro de la zona de estudio, en su mayoría correspondientes a especies implantadas o que actualmente crecen de forma espontánea en el área; 8 se compran en otras partes del país, principalmente en el norte (Formosa, Chaco y Santiago del Estero) y 2 provienen tanto de la extracción como de la compra (Tabla 1).

La leña que se extrae de la zona es considerada de mediana a mala calidad, ya que hace llama pero no buena brasa: "Todo lo que es para llama se consigue en la zona" (H., 67 años, masculino. City Bell, 2017), por tal motivo, eligen traer de otras zonas del país, para complementar especies de madera más dura.

De las especies que se obtienen de la zona, se observó que bajo un mismo nombre local se pueden incluir varias especies de un mismo género, como sucede con los "álamos" y los "pinos" ya que, como se mencionó antes, se junta lo que se encuentra independientemente de la calidad de la leña, y estas especies son todas consideradas de mala calidad o que sirven sólo para llama. Por otro lado, para el caso de los "eucaliptos", algunos entrevistados reconocen distintos tipos de acuerdo al color, la dureza y el peso de la madera y otras características del árbol (la altura, la forma de la copa, el tamaño, el color y la forma de las hojas, el tipo y persistencia de la corteza, entre otros): "eucalipto colorado" (Eucalyptus camaldulensis Dehnh), "eucalipto medicinal" (E. sideroxylon A. Cunn. ex Woolls) y "eucalipto blanco", "eucalipto amarillo" y "eucalipto globulus" (todos correspondientes a E. globulus Labill.) Otros entrevistados mencionaban simplemente "eucalipto" sin reconocer tipos, por lo que podría suponerse que se refiere a cualquiera de ellos (Fig. 4 y Tabla 2).

\section{Etnotaxones más vendidos}

Los más vendidos, según los entrevistados, son el "quebracho colorado", luego el "itín" y en tercer lugar el "eucalipto" (Eucalyptus spp.); la mayoría de los entrevistados no reconoce una especie en particular de eucalipto (Fig. 5). Asimismo, todos coinciden en señalar que las mejores especies para leña son el "quebracho colorado", el "itín" y el "eucalipto", este último no por su calidad sino porque es más barato: "El quebracho viene del norte, y el transporte es carísimo (...) es más cara, entonces la gente compra más eucalipto que quebracho, se llevan por ejemplo $150 \mathrm{~kg}$ de eucalipto y $100 \mathrm{de}$ quebracho. Además, el eucalipto, como hace buena llama, sirve para encender al quebracho que es muy duro y no prende fácil" (F. 60 años, femenino. City Bell, 2017).

El 46\% de los comercios, además, vende carbón de "quebracho blanco" o de "itín", proveniente de las provincias del Chaco o Santiago del Estero. Ambas especies son utilizadas para fabricar carbón, porque no hacen chispa al encenderlo.

\section{Uso de leña en sus hogares}

Sólo el 38\% de los entrevistados utiliza leña en su hogar con fines de calefacción y, en un único caso, para cocinar en estufa-hogar. "Yo tengo salamandra chica, uso $10 \mathrm{~kg}$ por día. Es caro, gasto 50 pesos por día sólo en calefacción. Pero no hay gas natural donde vivo" (F. 60 años, femenino. City Bell, 2017). Las especies que eligen los expendedores para su consumo son "quebracho colorado" y "eucalipto" (sin especificar) para encender, o la leña de mayor disponibilidad en la zona, como "álamo", "sauce" y "ciprés". El resto de los entrevistados no utiliza leña, porque prefieren el gas natural o la electricidad, excepto un caso en que no utiliza leña porque no dispone de salamandra ni hogar. 
Bol. Soc. Argent. Bot. 53 (3) 2018
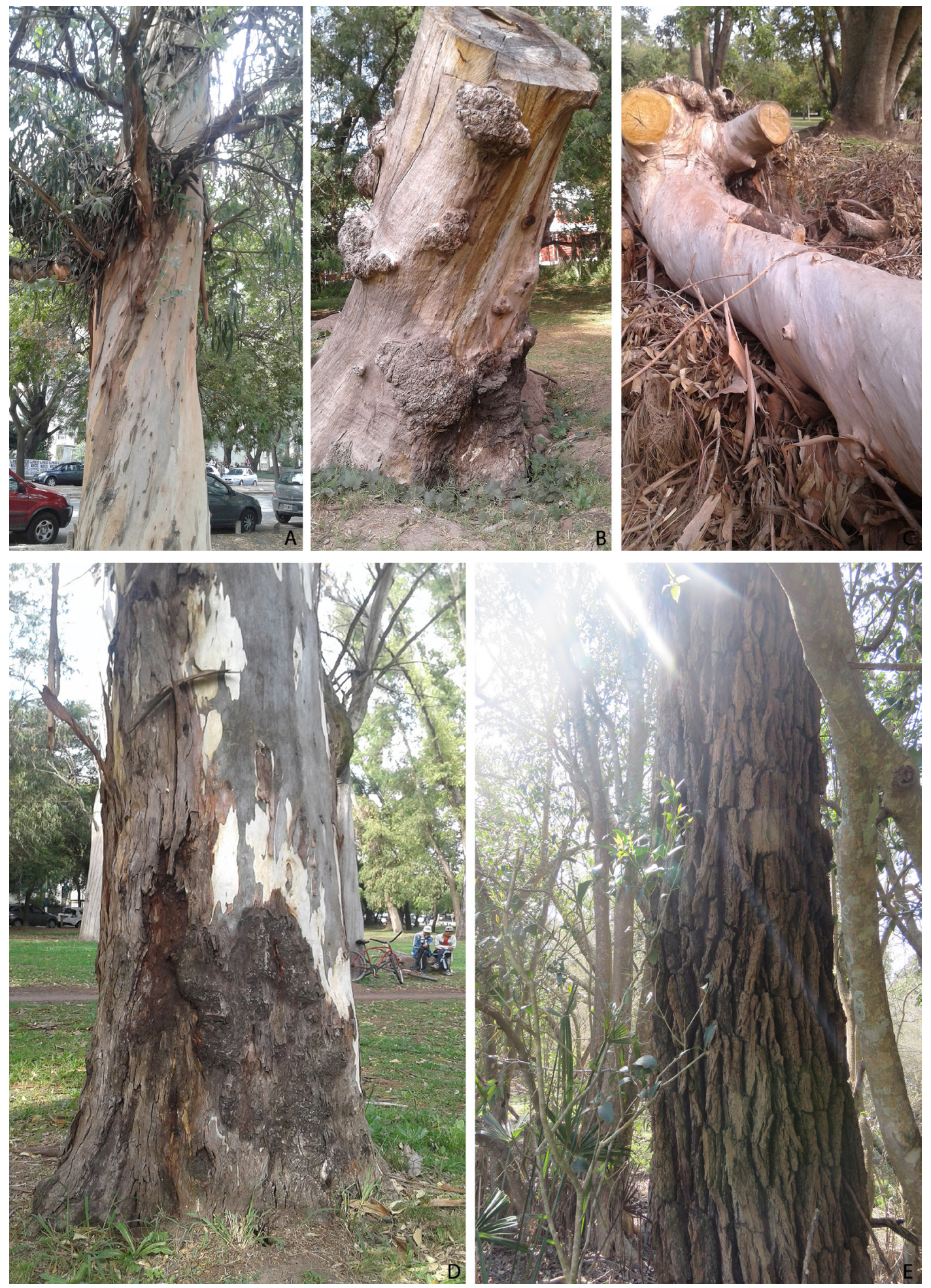

Fig 4. Etnoespecies y etnovariedades del género Eucalyptus. A: eucalipto blanco; B: eucalipto globulus, medicinal; C: eucalipto amarillo; D: eucalipto colorado; E: eucalipto medicinal. 
Tabla 2. Tipos de eucaliptos reconocidos localmente.

\begin{tabular}{|c|c|c|}
\hline Nombre local & Forma local de reconocimiento & $\begin{array}{l}\text { Nombre } \\
\text { científico }\end{array}$ \\
\hline $\begin{array}{l}\text { Eucalipto } \\
\text { colorado }\end{array}$ & $\begin{array}{l}\text { Es el más común y el mejor para leña. Cuando se seca, al no ser tan dura, se } \\
\text { parte por los radios. Color de la madera: colorada }\end{array}$ & $\begin{array}{l}\text { Eucalyptus } \\
\text { camaldulensis }\end{array}$ \\
\hline $\begin{array}{l}\text { Eucalipto } \\
\text { amarillo }\end{array}$ & $\begin{array}{l}\text { Crece con más altura, no tanto para los lados. Cáscara lisa y se desprende } \\
\text { quedando colgada. El tronco se retuerce, cuando se parte sale como en espiral, } \\
\text { es muy pesado y difícil de cortar. Color de la madera: amarilla. }\end{array}$ & $\begin{array}{l}\text { Eucalyptus } \\
\text { globulus }\end{array}$ \\
\hline Eucalipto blanco & $\begin{array}{l}\text { Alto. Tronco retorcido, blanco y pierde la cáscara, se pone más liso que el } \\
\text { colorado. Algunos son buenos como leña y otros no. Para hacer astillas conviene } \\
\text { cortarlo verde, seco no porque es durísimo. Algunos se ponen negros cuando lo } \\
\text { pones en la estufa. Color de la madera: blanca. }\end{array}$ & $\begin{array}{l}\text { Eucalyptus } \\
\text { globulus }\end{array}$ \\
\hline $\begin{array}{l}\text { Eucalipto } \\
\text { globulus }\end{array}$ & $\begin{array}{l}\text { Es pura cáscara y blando. Se le hacen como globos al tronco por eso le dicen } \\
\text { globulus. La leña es una de las más pesadas porque tiene mucha agua. Color de } \\
\text { la madera: blanca o amarilla-anaranjada. }\end{array}$ & $\begin{array}{l}\text { Eucalyptus } \\
\text { globulus }\end{array}$ \\
\hline $\begin{array}{l}\text { Eucalipto } \\
\text { medicinal }\end{array}$ & $\begin{array}{l}\text { Tiene cáscara gruesa y "peluda", como con canaletas, la hoja es mas plateada, } \\
\text { circular, media grisácea. Es bueno pero cuando se seca se tuerce y es difícil de } \\
\text { cortar. Color de la madera: blanca o amarilla. }\end{array}$ & $\begin{array}{l}\text { Eucalyptus } \\
\text { sideroxylon }\end{array}$ \\
\hline
\end{tabular}

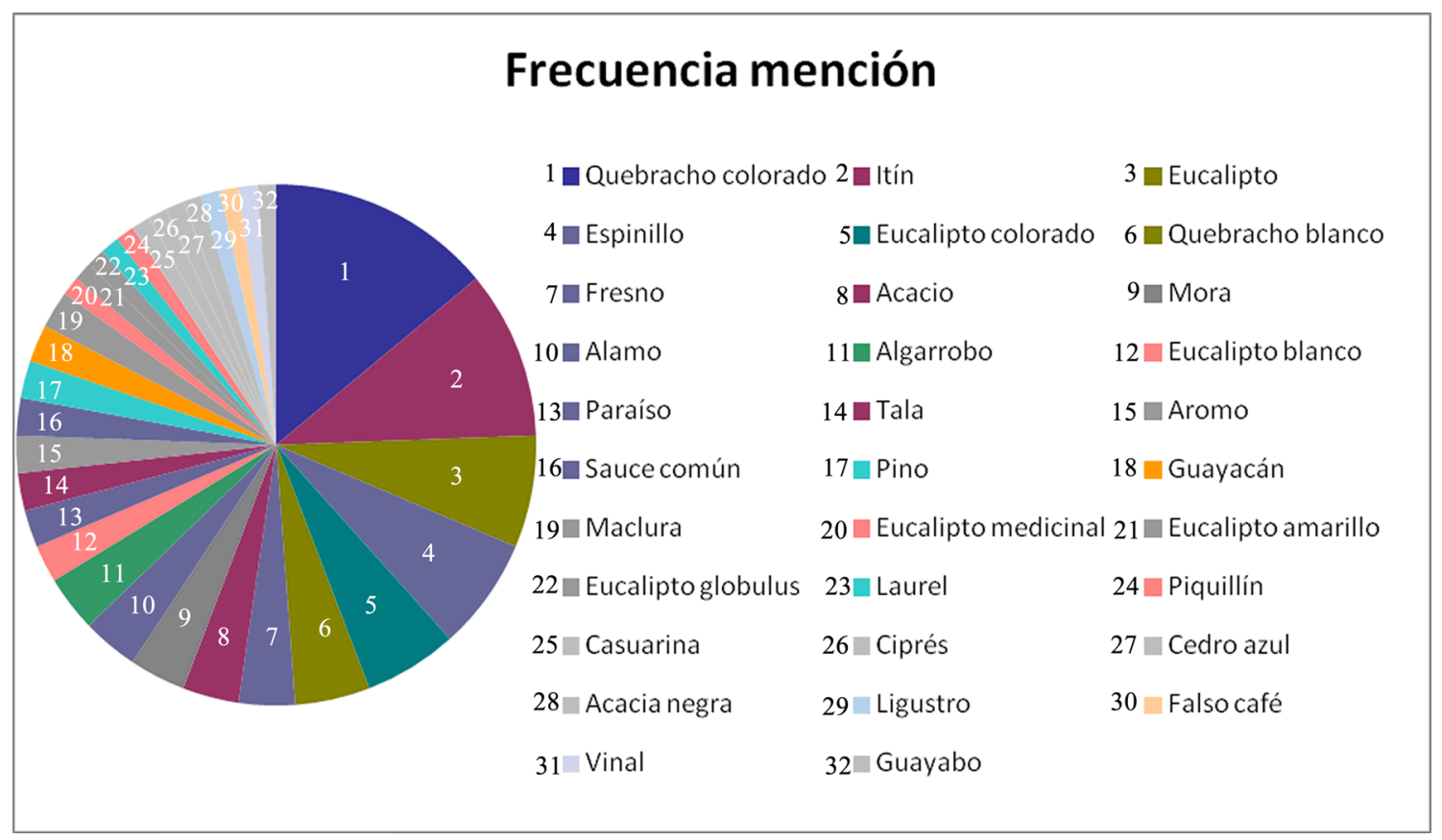

Fig. 5. Frecuencia de mención de etnotaxones presentes en el circuito comercial.

\section{Discusión Y CONCLUSIONES}

Se registraron 32 etnotaxones comercializados como leña en el partido de La Plata, 22 de ellos se extraen de la zona en estudio y en su mayoría corresponden a especies exóticas. El resto es comprado y traído para su reventa desde otras zonas del país (principalmente del norte argentino) y corresponden en su mayoría a especies nativas de su área de origen. Cabe destacar que la categoría nativo/exótico es utilizada a los fines prácticos de organizar la información pero no es significativa 
desde la visión del poblador local ya que no utiliza dicha clasificación.

En la Patagonia argentina, en la provincia de Chubut, Morales et al. (2017) relevaron 28 especies utilizadas como leña en poblados pequeños y zonas rurales, mientras que Arré et al. (2015), en la misma provincia, en un estudio etnobotánico urbano en la ciudad de Esquel, relevaron sólo 8 especies comercializadas. Por su parte, Cardoso et al. (2012, 2013), mencionan 27 especies empleadas por los pobladores rurales a nivel doméstico en la provincia de Río Negro. Según estos resultados, en el ámbito rural de la Patagonia argentina, hay una mayor cantidad de especies utilizadas como leña que en el ámbito urbano. Sin embargo, Stampella et al., (2016) en un estudio realizado en la región rioplatense donde se incluyen zonas rurales y suburbanas, hallaron 23 especies utilizadas como leña, en tanto que en el presente estudio realizado en el ámbito urbano, se halló un número considerablemente mayor. Más allá de la cantidad de especies aprovechadas como combustible, es evidente que el uso de las plantas y las interrelaciones plantas-humanos están moldeados por la historia, por los ambientes físicos y sociales y por las cualidades inherentes de las plantas mismas, es decir, por el estudio contextualizado del uso de las plantas (Pochettino et al., 2015).

En relación a las especies utilizadas como combustible, algunas coinciden con los resultados obtenidos por Cardoso et al. (2012, 2013), Arré et al. (2015) y Morales et al. (2017). Se trata de especies ampliamente cultivadas en la Argentina de los géneros Salix, Populus, Eucalyptus y Pinus (MAGyP, 2014), por lo que están disponibles para su uso tanto en la Patagonia como en la zona de estudio y son aprovechadas como leña por los pobladores aunque no son de las más valoradas.

Se destacan 3 etnotaxones por ser los más vendidos: el "quebracho colorado" y el "itín", debido a las características de sus maderas y el "eucalipto" debido a la relación precio/calidad. Si bien, en este estudio la especie más vendida, el "quebracho colorado", es además la preferida por los entrevistados, no se puede asumir que siempre sea de esta manera (Medeiros \& Albuqurque, 2011; Marofu et al., 1997).

En cuanto a la densidad, tanto el "quebracho colorado" como el "itín" tienen maderas de alta densidad, $\left(1,2 \mathrm{Kg} / \mathrm{dm}^{3}\right)$ en estado seco (Atencia, 2003). Por su parte, el "eucalipto" presenta una densidad media, es decir que su leña es bastante menos densa que los dos primeros, pero en relación con los sauces o álamos, es considerablemente mayor. Según estudios realizados por Cardoso (2013), la densidad de las maderas muestra una asociación significativa positiva con el Índice de valor combustible (IVC) por lo que se puede pensar que las especies de mayor densidad serán las que posean mayor IVC.

Las valoraciones y preferencia sobre la leña expresadas por los entrevistados coinciden con lo manifestado por Alves Ramos et al., (2008) quienes observan una relación directa entre la preferencia de las maderas y las características físicas de las mismas. A esto hay que agregar otra variable que es tenida en cuenta al momento de la compra de leña, según los entrevistados, como lo es el precio de la misma. Un ejemplo de ello, es el caso del "eucalipto", que representa una alternativa válida para aquellos usuarios que priorizan el precio por sobre la calidad de la leña.

$\mathrm{Si}$ bien en la zona no existen especies con densidades tan altas como las del "quebracho colorado" o el "itín", las especies que conforman el talar, principalmente el "tala", el "coronillo" y el "espinillo", poseen maderas que, debido a las cualidades que presentan, han sido utilizadas intensivamente desde comienzos de la ocupación hispánica bonaerense, en el siglo XVI (Athor, 2006; Torres Robles \& Tur, 2006), motivo por el cual se han visto reducidos y en el área donde los talares están mejores representados es en el PCS, donde su uso se encuentra restringido (Ordenanza N²94/98, Punta Indio) y por ende, las maderas de estas especies no pueden ser extraídas por los pobladores ni siquiera para uso doméstico. El "coronillo" no fue mencionado por ninguno de los entrevistados, probablemente porque no se encuentra disponible en la zona y crece fundamentalmente en la zona del PCS, por lo que no pueden acceder a él. El "tala", si bien hay mayor cantidad de ejemplares en la zona del PCS, crece además en algunos sectores cerca del partido de La Plata, principalmente en los partidos de Berisso y Ensenada, y sin embargo, solo unos pocos entrevistados lo mencionaron. Distinto es el caso del "espinillo", que se encuentra en el cuarto lugar de mención, es decir entre los más frecuentemente mencionados como leña. 


\section{B. Doumecq y P. M. Arenas - Conocimiento botánico local en leñeras de La Plata}

Éste, además de extraerse de la zona es traído para su venta desde la provincia de Entre Ríos. A las especies propias del talar hay que sumarle diversas especies implantadas y espontáneas, que poseen densidad media y son valoradas por algunos entrevistados y llamadas semiduras, como lo son la "acacia negra", el "fresno", la "acacia blanca", la "mora", la "casuarina"; otros, en cambio consideran que en la zona no hay maderas buenas para leña y solo sirven para llama por lo que deben comprar especies de madera dura de otras provincias de la Argentina.

El principal destino de la leña es para la calefacción de los hogares y, en segundo lugar, para cocinar, ya que para esta última se prefiere el uso de gas (natural o envasado). Se demuestra la vigencia del uso de leña en el ámbito urbano y periurbano aunque, de acuerdo con CIFOR, 2003, resulta que muchos de los entrevistados recurren a las plantas como combustible, más por necesidad que por elección. Algunos de ellos reconocieron que "deben caer" en el uso de leña porque en su hogar no disponen de gas natural (que es más económico que la leña o el gas envasado y probablemente porque es más cómodo, no hay que ir a buscarlo ni trasladarlo ni ocupa lugar como ocurre con la leña) mientras que más de la mitad de los entrevistados no utilizan leña en sus hogares aunque la tengan disponible porque prefieren el gas natural o la electricidad y ven a la venta de leña solo como una salida laboral.

Los entrevistados representan un grupo heterogéneo que albergan experiencias y saberes asociados a la actividad leñera. El caso de los "eucaliptos" refleja la diversidad de saberes que conviven en el área de estudio, ya que por un lado, hay quienes bajo ese nombre local incluyen a varias especies del género Eucalyptus sin distinguir entre ellas, mientras que otros entrevistados pueden identificar diferentes tipos de "eucaliptos" según las características del árbol, de su corteza, del color de la madera, la dureza, entre otras.

Se destaca el amplio conocimiento que poseen los entrevistados acerca de los productos que expenden en cuanto a la época en que debe cortarse la madera, el tiempo requerido de estacionado para que esté seca, el tipo de herramientas a utilizar, cuáles son las mejores especies para leña y cómo identificarlas, tanto en la planta en pie como a partir de la leña cortada.
Arré et al. (2015) señalan que la fuerte dependencia de uso circunscripto a un número restringido de especies pondría en serios riesgos la conservación de los recursos nativos. Sin embargo, en el área de estudio, esta situación no ocurre dado que se utiliza la madera de numerosas especies como leña y la mayoría de ellas son exóticas. De acuerdo con lo propuesto por Richeri et al. (2013) existen diferentes mecanismos y soluciones locales, donde se combinan los saberes previos con los cambios socio-ambientales. Ejemplo de ello es la incorporación de diversas especies exóticas de rápido crecimiento, muy abundantes en el partido de La Plata y alrededores como la "acacia negra", el "ligustro", la "mora", las que son aprovechadas por los vendedores por ser buenas para leña, tal y como sugiere Contreras-Hinojosa et al. (2003), quien propone la utilización de especies de rápido crecimiento para frenar el deterioro del bosque nativo.

Sin embargo, debido a que las dos especies más vendidas en el partido de La Plata como leña son el "quebracho colorado", una especie que ha sido muy explotada a lo largo del tiempo en su área de origen (Tortorelli, 1956), y el itín que su uso está prohibido en la Argentina (Ríos et al., 2001), es necesario discutir acerca de la continuidad del uso de estas especies que son utilizadas en todo el territorio argentino de manera irrestricta.

Este trabajo podría resultar de interés al momento de establecer estrategias tendientes a la conservación dado que, como se mencionó anteriormente, los saberes de los pobladores pueden ser de gran utilidad en la resolución de problemáticas ambientales locales tales como la identificación de estas especies valiosas como leña que resultan una alternativa al uso de plantas nativas.

\section{Agradecimientos}

A los pobladores que participaron en las entrevistas; a la Cátedra de Dendrología de la Facultad de Ciencias Agrarias y Forestales, UNLP, en particular a la Lic. Stella Rivera, a la Ing. Ftal. Emilce Galiussi y al Lic. Pablo Cabanillas, por permitirnos el acceso y consulta de la xiloteca, y la ayuda brindada. Al Dr. Julio A. Hurrell (Director de tesis de doctorado y beca de la Lic. Doumecq) por la lectura crítica del manuscrito y sus acertados comentarios. 


\section{Biblografía}

ALBUQUERQUE, U. P., M. ALVES RAMOS, R. F. P. LUCENA \& N. L. ALENCAR. 2014b. Methods and techniques used to collect ethnobiological data. In: ALBUQUERQUE, U. P., L. V. F. CRUZ DA CUNHA, R. F. P. LUCENA \& R. R. N. ALVES (eds.): Methods and techniques in Ethnobiology and Ethnoecology, pp. 15-37. Springer/Humana Press, New York.

ALBUQUERQUE, U. P. \& R. F. P. LUCENA. 2004. Métodos e técnicas na pesquisa etnobotânica. Livro Rápido-NUPEEA, Recife.

ALBUQUERQUE, U. P., R. F. P. LUCENA \& E. M. F. NETO. 2014a. Selection of research participants. In: ALBUQUERQUE, U. P., L. V. F. CRUZ DA CUNHA, R. F. P. LUCENA \& R. R. N. ALVES (eds.), Methods and techniques in Ethnobiology and Ethnoecology, pp. 1-13. Springer/Humana Press, New York.

ALEXIADES, M. 1996. Selected guidelines for ethnobotanical research: a field manual. The New York Botanical Garden, New York.

ALVES RAMOS, M. \& U. P. ALBUQUERQUE. 2012. The domestic use of firewood in rural communities of the Caatinga: How seasonality interferes with patterns of firewood collection. Biomass Bioenergy 39: 147-158.

ALVES RAMOS, M., P. MUNIZ DE MEDEIROS, A. L. SANTOS DE ALMEIDA, A. L. PATRIOTA FELICIANO \& U. P. ALBUQUERQUE. 2008. Can wood quality justify local preferences for firewood in an area of Caatinga (dryland) vegetation? Biomass Bioenergy 32: 503-509.

ARRÉ, J., S. MOLARES, A. LADIO \& A. KUTSCHKER. 2015. Etnobotánica de las plantas leñateras y su circuito comercial en una ciudad de la Patagonia argentina. Gaia Scientia 9: 41-48.

ATENCIA, M. E. 2003. Densidad de maderas (Kg/m3) ordenadas por nombre común. INTI - CITEMA. Disponible en: https:// www.inti.gob.ar/maderaymuebles/index. php?seccion=informaciontecnica [Acceso: 02 Mayo 2018].

ATHOR, J. 2006. Referencias bibliográficas históricas que delatan la presencia del talar en la ciudad de Buenos Aires. In: MÉRIDA, E. \& J. ATHOR, eds., Talares bonaerenses y su conservación, pp. 218222. Fundación de Historia Natural Félix de Azara, Buenos Aires.

BARSKY, A. 2005. El periurbano productivo, un espacio en constante transformación. Introducción al estado del debate, con referencias al caso de Buenos Aires. Scripta Nova 9: 36. Disponible en: http://www. ub.edu/geocrit/sn/sn-194-36.htm [Acceso: 02 Enero 2018].
BARSKY, A. 2010. La agricultura de "cercanías" a la ciudad y los ciclos del territorio periurbano. Reflexiones sobre el caso de la Región Metropolitana de Buenos Aires. En: A. SVETLIZA DE NEMIROVSKY (ed.), Agricultura periurbana en Argentina y globalización. Escenarios, recorridos y problemas, pp. 15-29. FLACSO, Buenos Aires.

BERNARD, R. H. 2000. Social research methods. Qualitative and quantitative approaches. California. Sage Publications, Thousand Oaks.

BURKART, R., N. O. BÁRBARO, R. O. SÁNCHEZ \& D. A. GÓMEZ. 1999. Eco-regiones de la Argentina. Buenos Aires, Administración de Parques Nacionales.

CABRERA, A. L. 1971. Fitogeografía de la República Argentina. Bol. Soc. Argent. Bot. 14: 1-42.

CARDOSO, M. B., 2013. Utilización de especies combustibles en comunidades locales del noroeste de Patagonia: Bienes culturales y ambientales en la subsistencia rural. Tesis Doctoral. Centro Regional Universitario Bariloche, Universidad Nacional del Comahue.

CARDOSO, M. B., A. H. LADIO, S. M. DUTRUS \& M. LOZADA. 2015. Preference and calorific value of fuelwood species in rural populations in northwestern Patagonia. Biomass Bioenergy 81: 514-520.

CARDOSO, M. B., A. H. LADIO \& M. LOZADA. 2012. The use of firewood in a Mapuche community in a semi-arid region of Patagonia, Argentina. Biomass Bioenergy 46: 155-164.

CARDOSO, M. B., A. H. LADIO \& M. LOZADA. 2013. Fuelwood consumption patterns and resilience in two rural communities of the northwest Patagonian steppe, Argentina. J. Arid. Environ. 98: 146-152.

CIFOR (CENTER FOR INTERNATIONAL FORESTRY RESEARCH). 2003. Fuelwood and the poor. CIFOR Annu. Rep. 2003: 12-13. Disponible en: http:/www.cifor.org/publications/ pdf_files/AReports/AReport03s.pdf. [Acceso: 02 Enero 2018].

CIFOR (CENTER FOR INTERNATIONAL FORESTRY RESEARCH). 2012. Forests, fuel wood and charcoal. CIFOR Annu. Rep. 2012. Disponible en: https://www.cifor.org/publications/ pdf_files/factsheet/4063-factsheet.pdf. [Acceso: 02 Enero 2018].

CONTRERAS-HINOJOSA, J. R., V. VOLKEHALLER, J. L. OROPEZA-MOTA, C. RODRÍGUEZ-FRANCO, T. MARTÍNEZSALDAÑA \& Á. MARTÍNEZ-GARZA. 2003. Disponibilidad y uso de leña en el municipio de Yanhuitlán, Oaxaca. Terra 21: 437- 445. 
DELUCCHI, G., A. A. JULIANELLO \& R. F. CORREA. 1993. Los espacios verdes y el arbolado urbano en el área de La Plata. I. Orígenes y evolución hasta el presente. Museo 1: 61-65.

GHIANI ECHENIQUE, N, M. B. DOUMECQ \& M. L. POCHETTINO. 2018. Saberes botánicos en el talar. Utilización de plantas silvestres con fines medicinales y alimenticios en el Parque Costero del Sur (partidos de Magdalena y Punta indio, Buenos Aires, República Argentina). Gaia Scientia 12: 56-80.

HURRELL, J. A. 2014. Urban Ethnobotany in Argentina: Theoretical advances and methodological strategies. Ethnobiol. Conserv. 3: 2, doi:10.15451/ec2014-6-3.3-1-11.

HURRELL, J. A. \& M. L. POCHETTINO. 2014. Urban Ethnobotany: theoretical and methodological contributions. In: ALBUQUERQUE, U. P., L. V. F. CRUZ DA CUNHA, R. F. P. LUCENA \& R. R. N. ALVES (eds.), Methods and techniques in Ethnobiology and Ethnoecology, pp. 293-309. Springer/Humana Press, New York.

HURRELL, J. A., E. A. ULIBARRI, J. P. PUENTES, F. BUET COSTANTINO, P. M. ARENAS, \& M. L. POCHETTINO. 2011. Leguminosas medicinales y alimenticias utilizadas en la conurbación Buenos Aires-La Plata, Argentina. Bol. Lat. Caribe Pl. Med. Aromat. 10: 443-455.

INDEC. 2010. Instituto Nacional de Estadística y Censos. Censo Nacional de Población, Hogares y Viviendas. Disponible en: https://www.indec. gov.ar/censos_total_pais.asp?id_tema_1 $=2 \& i d$ tema_2 $=41 \& \mathrm{id}$ _tema_3 $=135 \& \mathrm{t}=\overline{0} \& \mathrm{~s}=0 \overline{\mathrm{d}} \mathrm{c}=2010$. [Acceso: 3 Enero 2018].

LEONARDIS, J. 1977. Libro del árbol. Tomo III. Esencias Forestales no Autóctonas Cultivadas de Argentina de Aplicación Ornamental y/o Industrial. Celulosa Argentina, Buenos Aires.

MAGyP (MINISTERIO DE AGRICULTURA, GANADERÍA Y PESCA). 2014. Argentina: plantaciones forestales y gestión sostenible. Disponible en: http://forestoindustria.magyp.gob. ar/archivos/gestion-forestal-sostenible/publi ambiental.pdf. [Acceso: 10 de mayo, 2018].

MAROFU, M., J. LUDWIG, M. O. ANDREAE, F. X. MEIXNER \& G. HELAS. 1997. Domestic biomass burning in rural and urban Zimbabwe. Biomass Bioenergy 12: 53-68.

MARTIN, G. 1995. Ethnobotany. A methods manual. London, Chapmann \& Hall.

MEDEIROS, P. M. \& U. P. ALBUQUERQUE. 2011. Methods to study the ethnobotany of wood resources: a critical evaluation. Bioremediation, Biodivers. Bioavail. 5: 14-21.
MORALES, D. V, S. MOLARES \& A. H. LADIO. 2017. Firewood Resource Management in Different Landscapes in NW Patagonia. Front. Ecol. Evol. 5: 111.

OGUNKUNLE, A. T. J. \& F. A. OLADELE, 2004. Ethnobotanical study of fuelwood and timberwood consumption and replenishment in Ogbomoso, Oyo state, Nigeria. Environ. Monitor. Assess. 91: 223236.

PARODI, L. R. 1940. Distribución geográfica de los talares de la Provincia de Buenos Aires. Darwiniana 4: 33-56.

POCHETTINO, M. L., P. M. ARENAS, D. SÁNCHEZ \& R. CORREA. 2008. Conocimiento botánico tradicional, circulación comercial y consumo de plantas medicinales en un área urbana de Argentina. Bol. Lat. Caribe Pl. Med. Aromat. 7: 141-148.

POCHETTINO, M. L., J. A. HURRELL \& M. M. BONICATTO. 2014. Horticultura periurbana: estudios etnobotánicos en huertos familiares y comerciales de la Argentina. Ambienta (España) 107: 86-99.

POCHETTINO, M. L., D. A. LAMBARÉ., P.C. STAMPELLA, M.B. DOUMECQ \& N. GHIANI ECHENIQUE. 2015. Especies arbóreas como "texto" en contextos pluriculturales... La conservación como pretexto. Actas Reunión Antropología MERCOSUR, 2015. Disponible en: http://xiram.com.uy/actas-del-congreso/grupos-detrabajo/ponencias-grupo-de-trabajo-83. [Acceso: 19 Febrero 2018].

POCHETTINO, M. L., M. C. PALEO, M. M. PAEZ, M. B. DOUMECQ \& N. GHIANI ECHENIQUE. En prensa. Dos mil años de historia del litoral bonaerense relatados por el tala. Abordaje interdisciplinar del Celtis ehrenbergiana (Klotzsch) Liebm. como patrimonio biocultural a través del tiempo en el Parque Costero del Sur (partidos de Magdalena y Punta Indio, provincia de Buenos Aires, República Argentina). Actas VI Congreso Internacional Etnobotánica, Córdoba 2014, España.

RICHERI, M. M. B. CARDOSO \& A. H. LADIO. 2013. Soluciones locales y flexibilidad en el conocimiento ecológico tradicional frente a procesos de cambio ambiental: estudios de caso en Patagonia. Ecol. Austral 23: 184-193.

RÍOS, N. A., A. M. GIMÉNEZ \& J. G. MOGLIA. 2001. Crecimiento del itín (Prosopis kuntzei Harms) en la región chaqueña argentina. Madera y Bosques 7: 47-56.

RIVERA, S. M. \& E. GALIUSSI. 2015. Identificación De Maderas Comerciales: Técnicas, Certificación de Identidad y Pericias. Libro de Cátedra Volumen I. Cátedra de Dendrología, Facultad de Ciencias Agrarias y Forestales, U.N.L.P. 
STAMPELLA, P. C, M. B. DOUMECQ, M. VOJKOVIC \& L. LABORDA. 2016. Valoración del cambio ambiental según los junqueros y leñateros en el sector sur de la región rioplatense (Argentina). Bonplandia 25: 17-31.

TORRES ROBLES, S. 2009. Variación geográfica de la composición y riqueza de plantas vasculares en los talares bonaerenses y su relación con el clima, sustrato, estructura del paisaje y uso. 2 vol. Tesis doctoral. Facultad de Ciencias Naturales y Museo. Universidad Nacional de La Plata.

TORRES ROBLES, S. S. \& N. M. TUR (2006). Los talares de la provincia de Buenos Aires. En: BROWN, A., U. MARTÍNEZ ORTIZ, M. ACERBI
\& J. CORCUERA, eds., La situación ambiental argentina 2005, pp. 246-250. Fundación Vida Silvestre Argentina. Buenos Aires.

TORTORELLI, L. A. 1956. Maderas y bosques argentinos. 1ra. ed. ACME, Buenos Aires.

Recibido el 28 de febrero de 2018, aceptado el 25 de junio de 2018. Editora: Norma I. Hilgert. 